\title{
Vortex Interactions and Barotropic Aspects of Concentric Eyewall Formation
}

\author{
HuNG-CHI KuO \\ Department of Atmospheric Sciences, National Taiwan University, Taipei, Taiwan \\ WAYNE H. SCHUBERT \\ Department of Atmospheric Science, Colorado State University, Fort Collins, Colorado \\ Chia-Ling Tsai and Yu-Fen Kuo \\ Department of Atmospheric Sciences, National Taiwan University, Taipei, Taiwan
}

(Manuscript received 13 September 2007, in final form 18 April 2008)

\begin{abstract}
Concentric eyewall formation can be idealized as the interaction of a tropical cyclone core with nearby weaker vorticity of various spatial scales. This paper considers barotropic aspects of concentric eyewall formation from modified Rankine vortices. In this framework, the following parameters are found to be important in concentric eyewall formation: vorticity strength ratio, separation distance, companion vortex size, and core vortex skirt parameter. A vorticity skirt on the core vortex affects the filamentation dynamics in two important ways. First, the vorticity skirt lengthens the filamentation time, and therefore slows moat formation in the region just outside the radius of maximum wind. Second, at large radii, a skirted core vortex induces higher strain rates than a corresponding Rankine vortex and is thus more capable of straining out the vorticity field far from the core. Calculations suggest that concentric structures result from binary interactions when the small vortex is at least 4-6 times as strong as the larger companion vortex. An additional requirement is that the separation distance between the edges of the two vortices be less than 6-7 times the smaller vortex radius. Broad moats form when the initial companion vortex is small, the vorticity skirt outside the radius of maximum wind is small, and the strength ratio is large. In concentric cases, an outer vorticity ring develops when the initial companion vortex is large, the vorticity skirt outside the radius of maximum wind is small, and the strength ratio is not too large. In general, when the companion vortex is 3 times as strong as the core vortex and the separation distance is $4-6$ times the radius of the smaller vortex, a core vortex with a vorticity skirt produces concentric structures. In contrast, a Rankine vortex produces elastic interaction in this region of parameter space. Thus, a Rankine vortex of sufficient strength favors the formation of a concentric structure closer to the core vortex, while a skirted vortex of sufficient strength favors the formation of concentric structures farther from the core vortex. This may explain satellite microwave observations that suggest a wide range of radii for concentric eyewalls.
\end{abstract}

\section{Introduction}

The general interaction of two vorticity patches with the same vorticity, but with different horizontal sizes and separation distances, has been described by Dritschel and Waugh (1992, hereinafter DW). Based on a quantification of the final to initial circulation of each vortex, the vortex interaction can be classified as one of

Corresponding author address: W. H. Schubert, Department of Atmospheric Science, Colorado State University, Fort Collins, CO 80523.

E-mail: waynes@atmos.colostate.edu five types: elastic interaction, partial straining out, complete straining out, partial merger, and complete merger. Many observed cases of tropical cyclone interactions resemble the idealized simulations of DW (e.g., see Larson 1975; Lander and Holland 1993; Kuo et al. 2000; Khain et al. 2000; Prieto et al. 2003). In the complete straining-out regime the smaller vortex is drawn out into a thin, tightly wound filament of vorticity surrounding the larger vortex, with no incorporation of fluid into the larger vortex. This bears a certain resemblance to secondary eyewall structures, although the model concentric structure has too much finescale filamentation compared to observed secondary eye walls. 
Observations of Typhoon Lekima at 0935 LT 25 September 2001 (see Fig. 1 of Kuo et al. 2004, hereafter $\mathrm{KLCW}$ ) indicate that it had a large area of convection with weak cyclonic vorticity outside the core vortex. Within approximately $10 \mathrm{~h}$, this weak vorticity wrapped around the inner eyewall. This scenario can be idealized as the binary interaction of a small, strong vortex (the tropical cyclone core) with a large, weak vortex (the vorticity induced by moist convection outside the core vortex). The DW analysis does not include this type of binary interaction. Their vortices were assumed to have the same strength, and thus their larger vortex was always the "victor." More realistic simulations of tropical cyclone merger and concentric eyewall formation (KLCW) have been obtained by relaxing the assumption that the interacting patches have the same vorticity. The introduction of the "vorticity strength ratio" adds a third dimension to the Dritschel-Waugh parameter space. As a result, binary interactions manifest two new stable end states: concentric vorticity structure and tripole vorticity structure. Both appear relevant for tropical cyclones. A tripole consists of three regions of alternately signed vorticity arranged in a line that rotates steadily in the same sense as the vorticity of the elliptically shaped central core (Carton et al. 1989; Polvani and Carton 1990; Carton and Legras 1994; Kloosterziel and Carnevale 1999). Examples of elliptical eyes that might be associated with tripolar vorticity structure have been reported by Kuo et al. (1999) for the case of Typhoon Herb (1996) and by Reasor et al. (2000) for the case of Hurricane Olivia (1994).

In a concentric eyewall, both the deep convection and the potential vorticity (PV) are enhanced (e.g., see the Hurricane Gilbert example given in Fig. 1 of Kossin et al. 2000). In terms of PV dynamics, the formation of a concentric eyewall has two important aspects: (i) an existing asymmetric PV distribution is organized by horizontal advective processes and (ii) the PV is diabatically enhanced during the organizational process. Although both aspects are probably important, it is useful to isolate aspect (i) through the study of highly idealized dynamical models. In this spirit, previous researchers have used nondivergent barotropic models to elucidate this aspect (KLCW; Rozoff et al. 2006, hereafter RSMK). The results of KLCW suggest that the formation of a concentric vorticity structure requires that the core vortex possess vorticity at least 6 times stronger than the neighboring vorticity patch, that the neighboring vorticity area be considerably larger than the core vortex, and that the separation distance between the core vortex and the neighboring vorticity patch be less than 3-4 times the core vortex radius.
Figure 1 shows passive microwave views of four western Pacific Ocean typhoons with concentric eyewalls. ${ }^{1}$ In these examples a large area of deep convection (brown) outside the core vortex appears to wrap around the inner eyewall to form a concentric eyewall on a time scale of approximately $12 \mathrm{~h}$. These cases are in general agreement with the Typhoon Lekema observations. The initial separation distance between the outer deep convection region and the vortex core varies from case to case. For example, the outer deep convection almost touched the vortex core in Typhoon $\mathrm{Du}$ juan, while in Typhoon Imbudo (Maemi) the deep convection lay approximately $50 \mathrm{~km}(100 \mathrm{~km})$ away from the vortex core. Typhoon Winnie possessed a huge area of deep convection some $260 \mathrm{~km}$ south of the core vortex, with a primary elongated band surrounding the core vortex. At later times, concentric eyewalls developed at radii roughly corresponding to the initial separation distances. In particular, the largest concentric eyewall was found in Typhoon Winnie, with an outer eyewall radius of $275 \mathrm{~km}$ (Zhang et al. 2005). The ratio of Winnie's moat width to core radius is 6 . The preponderance of large-radius concentric eyewalls in the west Pacific relative to other basins is discussed by Hawkins et al. (2006).

To explain vortex interaction over such a large distance, the previous binary vortex interaction results of KLCW must be extended. They only studied vortices with sharp vorticity edges (i.e., unskirted vortices), and, in such vortices, angular velocity decreases rapidly with radius outside the core. However, Mallen et al.'s (2005) aircraft flight-level data analysis shows that tropical cyclones are often characterized by a relatively slow decrease of tangential wind outside the radius of maximum wind (RMW), and hence by a corresponding cyclonic vorticity skirt. Vorticity skirts play several roles in tropical cyclone dynamics. For example, the outer vorticity gradients associated with each vortex may induce a vortex merger because of the enhanced propagation of each vortex (DeMaria and Chan 1984). The radial vorticity gradient associated with the vorticity skirt provides a background for the radial propagation of vortex Rossby waves (Montgomery and Kallenbach 1997; Balmforth et al. 2001). It also makes the vortex more resilient to the destructive effects of large-scale vertical wind shear (Reasor et al. 2004). More recently, it has been shown (Terwey and Montgomery 2008) that a radial vorticity gradient (or effective beta), along with a root-mean-square eddy velocity, provide a "Rhines

\footnotetext{
${ }^{1}$ These images are from the Naval Research Laboratory Marine Meteorology Division in Monterey, California (Hawkins et al. 2001).
} 


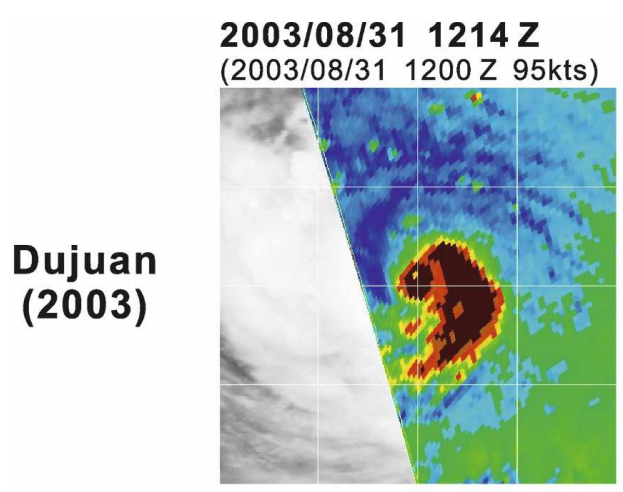

2003/08/312235 Z

(2003/09/01 0000 Z 120kts)

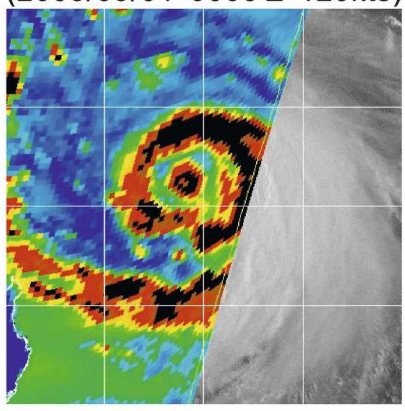

2003/07/20 $0942 \mathrm{Z}$

(2003/07/20 1200 Z 130kts)

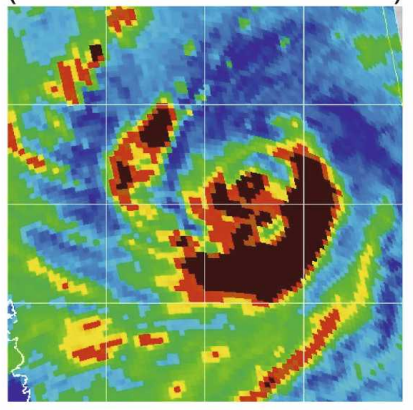

Imbudo

(2003)

Maemi

\section{3/09/09 2209 Z}

(2003/09/10 0000 Z 150kts)

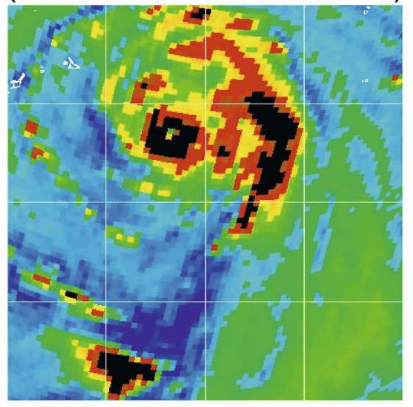

$1997 / 08 / 141031 \mathrm{Z}$ (1997/08/14 1200 Z 110kts)

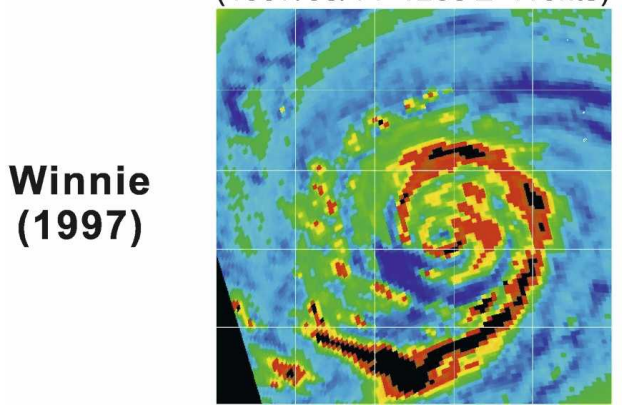

\section{Winnie} (1997)

(2003)

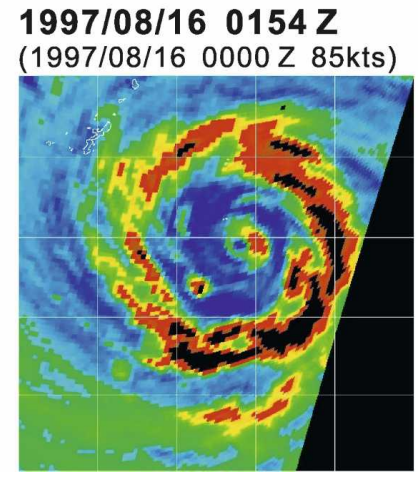

2003/07/20 2219 Z

(2003/07/21 0000 Z 130kts)

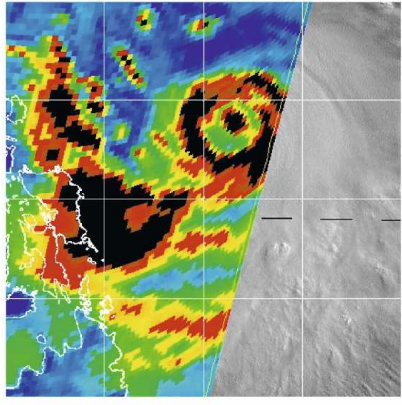

\section{3/09/10 $0925 Z$}

(2003/09/10 1200 Z 150kts)

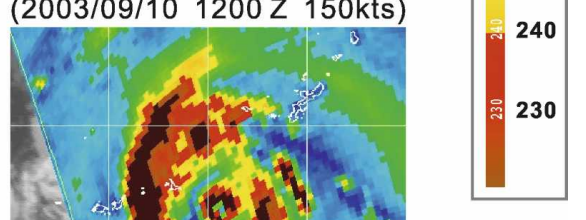

FIG. 1. Passive microwave image sequences for four western Pacific typhoons with concentric eyewalls (Dujuan, Imbudo, Maemi, and Winnie). For each typhoon the time interval is approximately $12 \mathrm{~h}$, and the estimated maximum winds are indicated at the top of the image. 
effect" for the anisotropic upscale cascade of eddy energy, which leads to the formation of a lower-tropospheric jet outside the primary eyewall. Here we shall illustrate how a skirt on a core vortex provides an additional circulation that leads to the formation of bands at large distances from the core and how the slower radial decrease of angular velocity associated with the vorticity skirt lengthens the filamentation time and slows moat formation.

This paper focuses on the role vorticity skirts play in the formation of concentric vorticity structures through binary vortex interaction. In particular, we explain why there is such a wide range of radii for concentric eyewalls. Section 2 describes the model, the solution method, the physical parameters, and the binary vortex interaction experiments. The filamentation dynamics of a cyclone core with a vorticity skirt are discussed in section 3 . The numerical results for binary vortex interactions with a skirted core vortex are presented in section 4 . Section 5 offers a summary and concluding remarks.

\section{Nondivergent barotropic model}

The basic dynamics considered here are two-dimensional, nondivergent, barotropic with ordinary diffusion, namely,

$$
\frac{\partial \zeta}{\partial t}+\frac{\partial(\psi, \zeta)}{\partial(x, y)}=\nu \nabla^{2} \zeta, \text { where } \quad \nabla^{2} \psi=\zeta
$$

is the invertibility principle relating the streamfunction $\psi$ and the vorticity $\zeta, \partial(\cdot, \cdot) / \partial(x, y)$ is the Jacobian operator, and $\nu$ is the constant diffusion coefficient. We perform calculations on a square domain and assume $\psi$ and $\zeta$ are doubly periodic. The model discretization is based on the Fourier pseudospectral method, with $512 \times 512$ equally spaced collocation points on a 300 $\mathrm{km} \times 300 \mathrm{~km}$ domain. The code was run with a dealiased calculation of the quadratic nonlinear terms with $170 \times 170$ Fourier modes. Time differencing is accomplished via the fourth-order Runge-Kutta method with a 3-s time step. The diffusion coefficient, unless otherwise specified, was chosen to be $\nu=6.5$ $\mathrm{m}^{2} \mathrm{~s}^{-1}$. For the $300 \mathrm{~km} \times 300 \mathrm{~km}$ domain this value of $\nu$ gives an $e^{-1}$ damping time of $3.37 \mathrm{~h}$ for all modes having total wavenumber 170 , and a damping time of $13.5 \mathrm{~h}$ for modes having total wavenumber of 85 . Some of the experiments were repeated at increased resolution and/or with a larger domain size. From these experiments we conclude that the results shown here are insensitive to both domain size and resolution. Obviously, the use of such a highly idealized model precludes the simulation of a complete secondary eyewall

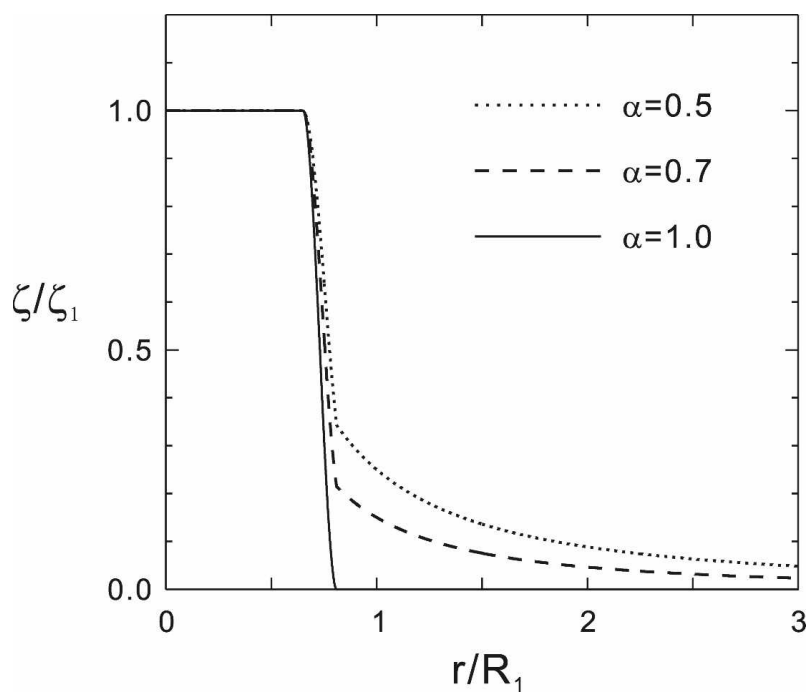

FIG. 2. Radial profiles of the vorticity for the strong core vortex with the skirt parameter a $\alpha=1.0,0.7$, and 0.5 .

cycle, but it does allow for some simple numerical experiments concerning the initial organizational processes involved in secondary eyewall formation.

The initial condition consists of two distinct vortices-a strong, skirted, core vortex and a weaker, larger, unskirted companion. The strong core vortex, denoted as vortex 1 , has the initial radial profile of vorticity shown in Fig. 2. This profile has constant vorticity $\zeta_{1}$ in the inner region, the skirted structure $(1 / 2) \zeta(1-\alpha)\left(r / R_{1}\right)^{-\alpha-1}$ in the outer region, with a cubic polynomial interpolation in the intermediate region $0.65 \leq r / R_{1} \leq 0.81$, where the constant $R_{1}$ is a measure of the core vortex size and $\alpha$ is the nondimensional skirt parameter. The constants in the cubic interpolating polynomial are determined in such a way that the vorticity and its radial derivative are continuous at the edges of the intermediate region. In the vorticity skirt region the azimuthal wind behaves as $r^{-\alpha}$. Aircraft observations of the azimuthal winds in hurricanes (e.g., Shea and Gray 1973; Mallen et al. 2005) suggest that a reasonable range for the skirt parameter is $0.5 \leq \alpha \leq 1$. Figure 2 depicts the initial vorticity of the stronger vortex for $\alpha=1.0,0.7$, and 0.5.

In contrast to the strong core vortex, the companion vortex is not skirted. This companion vortex, denoted as vortex 2 , has constant vorticity $\zeta_{2}$ near its center and zero vorticity outside the radius $R_{2}$. This vorticity structure is close to a step function but the edge has been smoothed to reduce the numerical Gibbs oscillations in the initial condition. We do not include a vorticity skirt on the companion vortex because our experience shows that the vorticity skirt on the "victorious" core vortex is more important. In addition, it is desirable to keep the number of parameters to a manageable level. 
$\mathrm{t}=\mathrm{Ohr}$

(a)

(b)

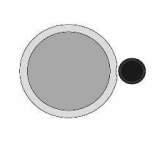

(c)

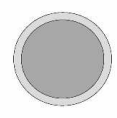

(d)
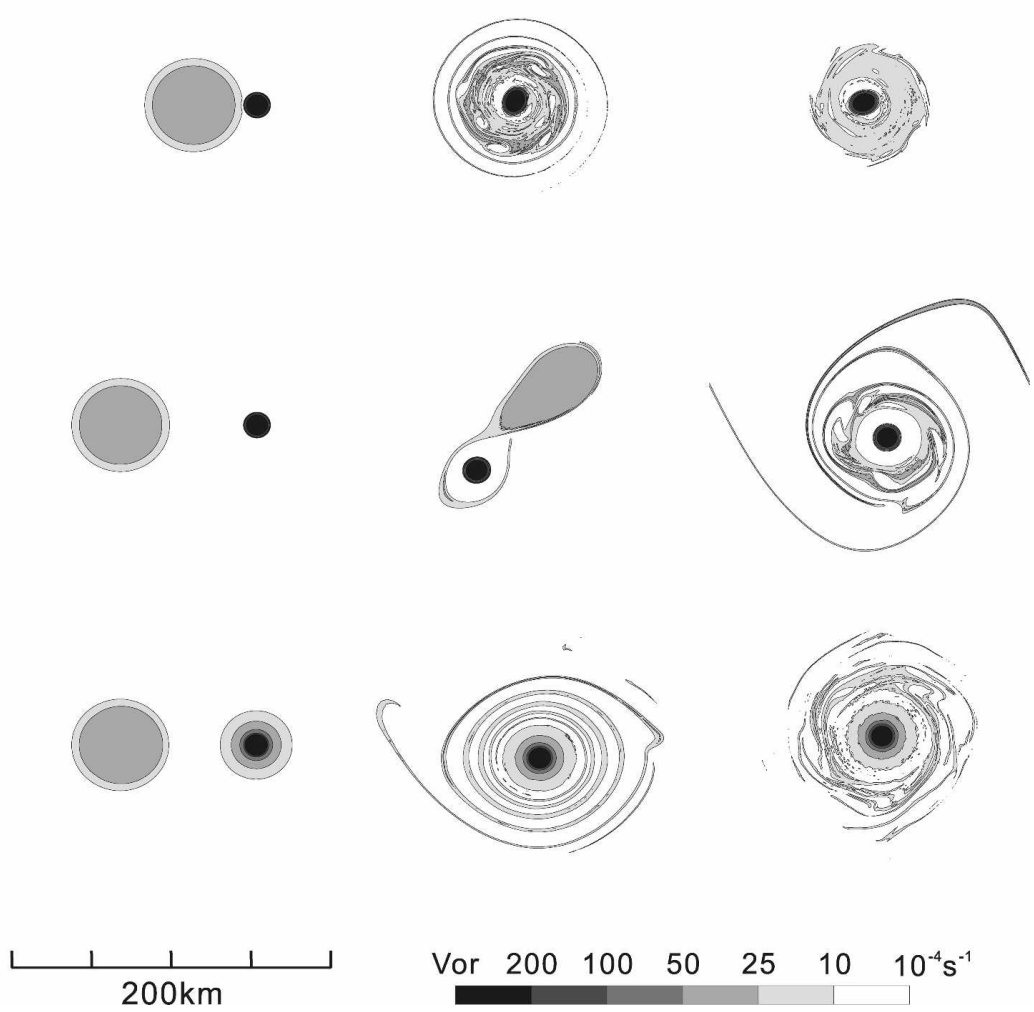

Vor $200 \quad 100 \quad 50 \quad 25 \quad 10 \quad 10^{-4} \mathrm{~s}^{-1}$

FIG. 3. Vorticity fields for the binary vortex experiments at hours 0,6 , and 12 , with $(\gamma, \alpha$, $\left.\Delta / R_{1}, r^{*}\right)=($ a) $(8,1,0,1 / 2)$; (b) $(8,1,0,1 / 4)$; (c) $(8,1,4,1 / 4)$; and (d) $(8,1 / 2,4,1 / 4)$.

Defining $d$ as the initial distance between the two vortex centers, we note that the initial condition contains the six parameters $\zeta_{1}, \zeta_{2}, R_{1}, R_{2}, d$, and $\alpha$. To set spatial and time scales to values representative of tropical cyclones, we choose the initial maximum vorticity of the companion vortex to be $\zeta_{2}=3 \times 10^{-3} \mathrm{~s}^{-1}$ and the "core size" of the intense skirted vortex to be $R_{1}=10$ $\mathrm{km}$. These choices reduce the number of parameters to four: the vorticity strength ratio $\gamma=\zeta_{1} / \zeta_{2}$, the vortex radius ratio $r^{*}=R_{1} / R_{2}$, the dimensionless gap $\Delta / R_{1}=$ $\left[d-\left(R_{1}+R_{2}\right)\right] / R_{1}$, and the skirt parameter $\alpha$. This paper investigates binary interaction for the following region of the parameter space: $1 \leq \gamma \leq 10,1 / 2 \leq r^{*} \leq$ $1 / 4,0 \leq \Delta / R_{1} \leq 7$, and $0.5 \leq \alpha \leq 1$. Using the initial condition described above, we have performed a set of experiments that illustrate the effects of a vorticity skirt.

\section{Filamentation dynamics of a cyclone core with a vorticity skirt}

Figure 3 shows the vorticity fields at hours 0,6 , and 12 for four experiments with the initial condition parameters listed in the figure caption. All four experiments produce concentric vorticity structures ${ }^{2}$ at hour 12 , but the range of behavior is striking. The initial condition for experiment $b$ is identical to experiment $a$, except that the size of the companion vortex has been increased, resulting in a wider outer band and a nar-

\footnotetext{
${ }^{2}$ In some ways, Fig. 3b resembles a tripolar vortex, but careful analysis of model vorticity fields around hour 12 leads us to classify it as a concentric vorticity structure. This case is similar to the observations and simulations of Oda et al. (2006).
} 
rower moat. ${ }^{3}$ The initial condition for experiment $\mathrm{c}$ is identical to $b$ except that the initial separation distance has been increased, resulting in a wide moat. The initial condition for experiment $\mathrm{d}$ is identical to experiment $\mathrm{c}$ except that the initial core vortex is now skirted, resulting in extreme wrapping at $6 \mathrm{~h}$ and a relatively chaotic concentric structure at $12 \mathrm{~h}$.

Concerning Figs. 3a,b, the wider outer band that evolves from the larger companion vortex is expected from the vorticity conservation point of view. The smaller moat size, however, may be understood from the filamentation dynamics. RSMK proposed the idea of a rapid filamentation zone outside the RMW of strong tropical cyclones. The rapid filamentation zone is a region of strain-dominated flow where the filamentation time is smaller than the 30 -min moist convective overturning time. Deep convection in the rapid filamentation zone may become highly distorted and even suppressed, leading to the formation of the moat. Purely nondivergent barotropic dynamics does not include convective effects, so the filamentation effect is similar to the stirring effect (e.g., Eckart 1948; Salmon 1998). Vorticity becomes strained out into thin filaments, which increases the vorticity gradient. The reduced spatial scale of vorticity elements and the increased vorticity gradient both enhance the mixing of the vorticity field. These filamentation dynamics may aid the formation of a moat. The filamentation time in the nondivergent barotropic framework, according to RSMK, is

$$
\tau_{\text {fil }}=2\left(S_{1}^{2}+S_{2}^{2}-\zeta^{2}\right)^{-(1 / 2)} \text { for } S_{1}^{2}+S_{2}^{2}>\zeta^{2},
$$

where $S_{1} / 2$ and $S_{2} / 2$ are the stretching deformation and the shearing deformation, respectively. According to (2), the filamentation time diminishes as $S_{1}^{2}+S_{2}^{2}$ increases. On the other hand, increased vorticity may offset the straining flow and lengthen the filamentation time. Figure $3 \mathrm{~b}$ illustrates these competing effects: the moat width shrinks because the straining flow is offset by the larger area of adjacent vorticity associated with the companion vortex.

Because of the larger width of the outer band, the size $^{4}$ of the concentric vorticity structure in Fig. $3 \mathrm{~b}$ is some $60 \%$ larger than that of the Rankine core vortex in Fig. 3a. By increasing the initial separation distance

\footnotetext{
${ }^{3}$ Here we use the term "moat" to denote the region of low vorticity between the high-vorticity core and the outer band consisting of vorticity values equal to $10^{-3} \mathrm{~s}^{-1}$, which is the same vorticity strength as the initial companion vortex.

${ }^{4}$ The size of a concentric vorticity structure is defined here as the total area covered by the core vortex, the skirt, the moat, and the outer band.
}

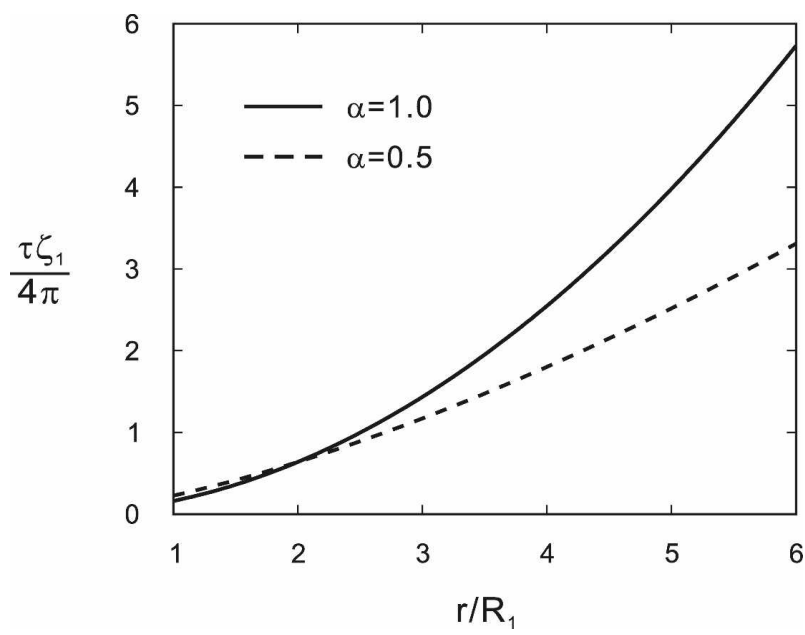

FIG. 4. The nondimensional filamentation time with respect to the core vortex eddy turn over time $4 \pi / \zeta_{1}$ as a function of the nondimensional radius $r / R_{1}$ for $\alpha=1$ and $\alpha=0.5$ core vortices.

and keeping all other parameters the same, the moat size increases, as can be seen by comparing Figs. 3b,c. Again, we attribute this to the fact that less vorticity is available outside the RMW when the initial separation distance of the companion vortex is large. The overall size of the concentric structures in Figs. 3b,c remain similar because of the thinness of the outer band in Fig. 3c.

We now examine the influence of a vortex skirt on $\tau_{\text {fill }}$. Outside the RMW the skirted core vortex has a total deformation given by

$$
S_{1}^{2}+S_{2}^{2}=\left(\frac{1+\alpha}{2}\right)^{2} \zeta_{1}^{2}\left(\frac{R_{1}}{r}\right)^{2+2 \alpha},
$$

which shows that the deformation is weaker for a skirted vortex (i.e., $\alpha<1$ ) in the region $r / R_{1} \leq 2$, and of comparable magnitude with the Rankine vortex in the far field. In addition, the skirted vorticity field outside the RMW may further modify the strain flow effect and the filamentation time. With the vorticity skirt, we have

$$
S_{1}^{2}+S_{2}^{2}-\zeta^{2}=\alpha \zeta_{1}^{2}\left(\frac{R_{1}}{r}\right)^{2+2 \alpha}
$$

so that the filamentation time in the skirt region is

$$
\tau_{\text {fil }}(r)=\frac{2}{\sqrt{\alpha} \zeta_{1}}\left(\frac{r}{R_{1}}\right)^{1+\alpha} .
$$

Figure 4 shows the nondimensional filamentation time (normalized with respect to the core vortex eddy turnover time $4 \pi / \zeta_{1}$ ) as a function of the nondimensional radius $\left(r / R_{1}\right)$ for the $\alpha=1$ Rankine vortex and the $\alpha=$ 0.5 skirted vortex. The filamentation time of the skirted 
vortex is slightly longer than that of a Rankine vortex in the region immediately outside the RMW, but far from the RMW $\left(5 \leq r / R_{1} \leq 6\right)$ its filamentation time is only $60 \%$ that of the Rankine vortex. This shorter far-field filamentation time is due to the presence of the additional circulation associated with the vorticity skirt. Thus, a skirted vortex is more capable of straining out the vorticity field far from its core. This explains why the moat size in the skirted vortex case (Fig. 3d) ${ }^{5}$ is smaller than that of the Rankine vortex (Fig. 3c), and why the moat in the skirted vortex case contains thin remnant vorticity filaments. It also explains why the outer band is thinner in the skirted vortex case. The size of the concentric structure in the skirted vortex in Fig. $3 \mathrm{~d}$ is some $25 \%$ larger than that from the Rankine vortex in Fig. 3c because of the larger radius of the skirted core.

Summarizing the above discussion on filamentation dynamics, the moat size is reduced when skirt vorticity lengthens the filamentation time in the region just outside the RMW. Conversely, the moat is broader when the initial companion vortex is small, the vorticity skirt outside the RMW is small, and the strength ratio is large. Finally, we note from Fig. 3 that pure advective dynamics are capable of producing a wide variation in the sizes of concentric vorticity structures. In particular, the concentric vorticity structure in Fig. $3 \mathrm{~d}$ is about twice as large as the one in Fig. 3a.

The selective decay principle of $2 \mathrm{D}$ turbulence theory (e.g., Matthaeus and Montgomery 1980; McWilliams 1984; Cushman-Roisin 1994) offers another way to explore the filamentation dynamics of a skirted vortex. Figure 5 gives the time dependence of total kinetic energy, enstrophy, and palinstrophy with respect to their initial values for the simulations shown in Figs. $3 c$,d. The scale for the palinstrophy (kinetic energy and enstrophy) is on the left (right) side of the figure. Figure 5 indicates that the total enstrophy

$$
Z=\iint \frac{1}{2} \zeta^{2} d x d y
$$

is selectively decayed over the total kinetic energy

$$
E=\iint \frac{1}{2} \nabla \psi \cdot \nabla \psi d x d y .
$$

This is a consequence of the fact that, according to the governing dynamics in (1), $E(t)$ and $Z(t)$ obey $d E / d t=$ $-2 \nu Z$ and $d Z / d t=-2 \nu P$, where

\footnotetext{
${ }^{5}$ The moat in the skirted vortex is the low vorticity region outside the vorticity skirt.
}

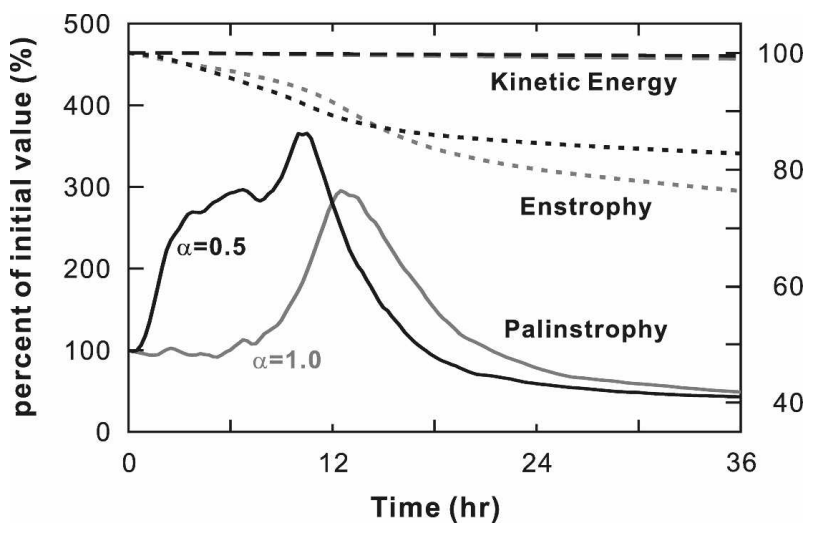

FIG. 5. Time dependence of kinetic energy, enstrophy, and palinstrophy (normalized by their respective initial values) for the simulations shown in Fig. $3 c(\alpha=1)$ and Fig. $3 d(\alpha=0.5)$. The scale for kinetic energy and enstrophy is on the right, and the scale for palinstrophy is on the left.

$$
P=\iint \frac{1}{2} \nabla \zeta \cdot \nabla \zeta d x d y
$$

is the total palinstrophy, a measure of the overall vorticity gradient. In the case of a nearly inviscid fluid (sufficiently small $\nu$ ) the vorticity contours can pack close together before diffusion or mixing is effective. The closely packed contours increase $|\nabla \zeta|$ and greatly enhance the palinstrophy. Even when $\nu$ is small, the $-2 \nu P$ term may not be small because of the increase of palinstrophy. This causes a significant enstrophy cascade, which leads to smaller enstrophy, and hence, a small $-2 \nu Z$ term. The result is that kinetic energy is nearly conserved.

The palinstrophy evolution shown in Fig. 5 indicates an initial increase and eventual decrease of the vorticity gradient for both cases. The evolution of $P$ is described by (e.g., Eckart 1948; Kossin and Schubert 2003)

$$
\begin{aligned}
\frac{d P}{d t}= & \iint\left[u_{x} \zeta_{x}^{2}+\left(v_{x}+u_{y}\right) \zeta_{x} \zeta_{y}+v_{y} \zeta_{y}^{2}\right] d x d y \\
& -\nu \iint\left(\nabla^{2} \zeta\right)^{2} d x d y
\end{aligned}
$$

The last term in (6) represents the effect of mixing, which always reduces the overall vorticity gradient in the domain. On the other hand, $P$ can rapidly increase because of the advective dynamics in the first term on the right-hand side of (6). For a small enough value of $\nu, P$ can surge to values much larger than its initial value. Note that $S_{1}^{2}+S_{2}^{2}-\zeta^{2}=-4 \partial(u, v) / \partial(x, y)$, and integration over the doubly periodic domain yields

$$
\iint \frac{1}{2}\left(S_{1}^{2}+S_{2}^{2}\right) d x d y=\iint \frac{1}{2} \zeta^{2} d x d y .
$$


Like enstrophy, the total rate of strain is selectively decayed. Thus, the surge of $P$ cannot continue forever-eventually $P$ must decrease because of the mixing.

The near conservation of kinetic energy and the damping of enstrophy lead to the selective decay phenomenon of two-dimensional turbulence. That is, over time, the vortices become larger and fewer (McWilliams 1984) through vortex merger processes. From our experiments, it appears that moat formation prevents the vortex from merging into a simple monopole. The evolution of $P$ (Fig. 5) also suggests that the stirring power of a skirted vortex is greater than that of a Rankine vortex. The width of the outer vorticity band is broader when the initial companion vortex is large, the vorticity skirt outside the RMW is small, and the strength ratio is not too large.

In summary, our analysis suggests that a vorticity skirt lengthens the filamentation time and slows moat formation in the region just outside the RMW. On the other hand, at larger radii, a skirted core vortex induces higher strain rates than a corresponding Rankine vortex and is thus more capable of straining out the vorticity field far from the core.

\section{Binary vortex interactions involving a skirted core vortex}

To elaborate on the discussion in section 3 , we now consider experiments with the skirt parameters $\alpha=0.7$ and $\alpha=0.5$. The vorticity in the skirt region of the core vortex may contribute significantly to the circulation at the radius of the companion vortex, especially when the skirt parameter $\alpha$ is small. For example, in the case $\alpha=$ 0.7 , the central region vorticity and the skirt region vorticity make approximately equal contributions to the circulation at a radius of $3 R_{1}$. In the case $\alpha=0.5$, the contribution from the skirt region is actually larger than the contribution from the central region.

Figure 6 shows vorticity fields at $t=12 \mathrm{~h}$ for binary interactions in which the dimensionless gap is fixed at $\Delta / R_{1}=3$. The first column depicts the initial condition. In the three right-hand columns the vortex strength ratio takes values of $\gamma=4,7$, and 10. In the rows of the figure, the vortex radius ratio takes values of $r^{*}=1 / 3$ and $1 / 4$, and the core vortex skirt parameter takes values of $\alpha=0.7$ (narrow skirt) and 0.5 (wide skirt). It is clear from Fig. 6 that the cases with $\alpha=0.7$ undergo behavior ranging from merger $(\gamma=4)$ to concentric vorticity structure with a moat $(\gamma=7$ and $\gamma=10)$. In the $\alpha=0.7$ cases, the moats are wider when the core vortex is stronger (high $\gamma$ ). On the other hand, the outer vorticity bands are thinner when the core vortex is strong. The outer bands broaden, however, when the initial companion vortex is large. In contrast to the diverse behaviors of the narrow skirt cases $(\alpha=0.7)$, all of the wide skirt cases $(\alpha=0.5)$ result in a merger. Figure 6 suggests that the core vorticity of the small vortex is crucial in the formation of concentric vorticity structures when the skirt is narrow $(\alpha=0.7)$.

Figure 7 is similar to Fig. 6 except that the dimensionless gap parameter has been increased to $\Delta / R_{1}=5$ The narrow skirt cases $(\alpha=0.7)$ now span a wide range of behaviors from partial straining out $\left(\gamma=4\right.$ and $r^{*}=$ $1 / 3)$, to complete straining out with very thin spiral bands $\left(\gamma=10\right.$ and $\left.r^{*}=1 / 3\right)$, to merger and/or tripole $\left(\gamma=4\right.$ and $\left.r^{*}=1 / 4\right)$, to concentric vorticity structure with a moat $\left(\gamma=4,7\right.$ and $r^{*}=1 / 3$ cases, $\gamma=7,10$ and $r^{*}=1 / 4$ cases $)$. The moats in the concentric vorticity cases shown in Fig. 7 are wider than the $\Delta / R_{1}=3$ cases shown in Fig. 6. The formation of the moat region in the $\alpha=0.7$ cases occurs through advection of the weak vorticity in the initial background vortex-free region. Cases with a larger initial separation distance (i.e., $\left.\Delta / R_{1}=5\right)$ allow larger moat widths than the $\Delta / R_{1}=3$ cases. The wide skirt cases $(\alpha=0.5)$ of sufficient strength (i.e., $\gamma=7$ ) allow concentric vorticity structures to form at a larger separation distance (i.e., $\left.\Delta / R_{1}=5\right)$ than previously.

For the concentric cases of Figs. 6 and 7, the outer vorticity band is broad when the initial companion vortex is large, the skirt is narrow, and the strength ratio is small (i.e., less straining-out effect far from the core). In fact, the $\alpha=0.5$ and $\gamma=10$ results shown in Fig. 7 resemble the straining-out regime of Dritschel and Waugh, with very thin outer bands apparent in both the $r^{*}=1 / 4$ and $r^{*}=1 / 3$ cases. The circular shapes of the outer bands in all these experiments highlight the important role of the core vortex in organizing and stabilizing the outer bands by the mechanisms discussed in Dritschel (1989) and Kossin et al. (2000). In particular, "adverse shear" dynamics is believed to play an important role in the stabilization process. This adverse shear effect on an outer band can be viewed as an external flow field associated with the core vortex flow outside its radius of maximum wind. This flow has the opposite sense as the flow produced by the outer band's vorticity alone, which tends to stabilize the outer band.

For the concentric vorticity structures in Fig. 7, moat widths hold steady for given values of $\gamma$ and $r^{*}$, but moat widths increase as $\gamma$ increases. Figure 7 suggests that a strong core vortex with a wide skirt $(\alpha=0.5)$ can form a concentric vorticity structure even when the initial companion vortex is located far away (e.g., $r^{*}=1 / 4$ and $\Delta / R_{1}=5$ ). However, it may also induce so much strain that the resulting structure resembles the strain- 

$\Delta / R_{1}=3 \quad t=0 h r$
$\mathrm{t}=12 \mathrm{hr}$
$\mathrm{t}=12 \mathrm{hr}$
$\mathrm{t}=12 \mathrm{hr}$
$\gamma=4$
$\gamma=7$
$\gamma=10$
$\underline{r^{*}=1 / 3}$
$\alpha=0.7$
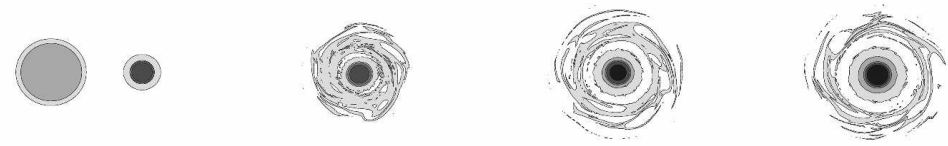
$\alpha=0.5$
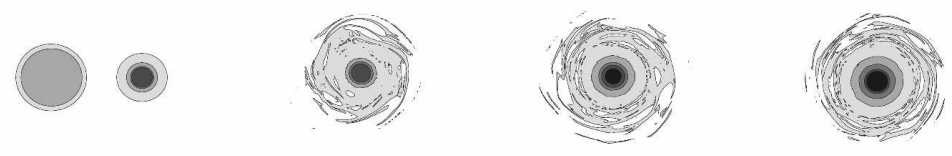
$\underline{r^{*}=1 / 4}$
$\alpha=0.7$
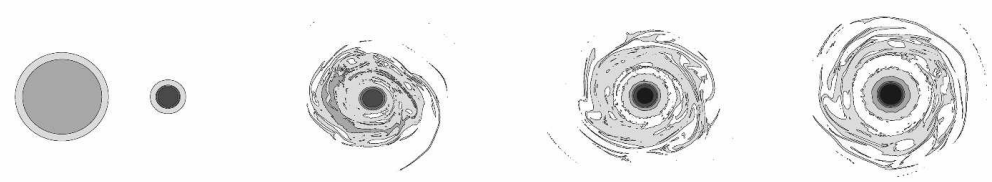
$\alpha=0.5$
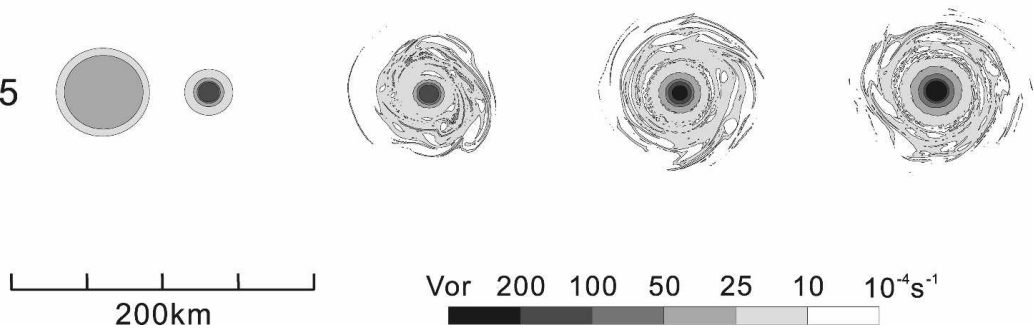
Vor $200 \quad 100 \quad 50 \quad 25 \quad 10 \quad 10^{-4} \mathrm{~s}^{-1}$

FIG. 6. Vorticity fields for binary vortex interactions at hour 12 with respect to the vorticity strength ratio $\gamma=\zeta_{1} / \zeta_{2}$ and the skirt parameter $\alpha$, with the dimensionless gap fixed at $\Delta / R_{1}=$ 3 , and with the vortex radius ratio $r^{*}=1 / 3$ (top two rows) and $r^{*}=1 / 4$ (bottom two rows). The first column depicts the initial condition.

ing-out regime. In contrast, the Rankine vortex $(\alpha=1)$ results of $\mathrm{KLCW}$ indicate that concentric structures only form when the separation distance is less than 3-4 times the core vortex radius. It appears that a core vortex with an extended vorticity gradient outside the RMW is capable of forming a concentric structure even when the initial outer vortex is large and located far from the core vortex. This result may be relevant for rare cases like Typhoon Winnie (see Fig. 1d), which had a concentric eyewall of very large diameter.

Figure 8 a shows the vorticity fields at $t=2,6$, and $12 \mathrm{~h}$ for the binary interaction case with $\alpha=0.7, \gamma=7$, $r^{*}=1 / 4$, and $\Delta / R_{1}=5$. During the first hour the large region of weaker vorticity becomes distorted into an approximately elliptical shape. As a result of the motion induced by its own vorticity, this ellipse turns counterclockwise, just as in the idealized Kirchhoff ellipse solution. Thus, one tip of the ellipse moves closer to the strong core vortex, while the other tip moves farther away-a process that conserves the angular impulse $\iint\left(x^{2}+y^{2}\right) \zeta d x d y$ (see the discussion in Melander et al. 1986). The part of the weaker vorticity region that is closest to the strong core vortex is then rapidly strained out and wound around the core vortex at close radius (see Fig. 8a at $t=2 \mathrm{~h}$ ). By $t=6 \mathrm{~h}$ the thin vorticity filaments have been wound several times, and by $t=$ $12 \mathrm{~h}$, vorticity mixing has created a halo of vorticity around the core vortex. If such an event were to occur in a real hurricane, and if it were observed by only a few radial legs of a research aircraft, the asymmetric nature of its early evolution might be lost, in which case it could be interpreted as an essentially axisymmetric contraction of a secondary wind maximum. For example, Fig. $8 \mathrm{~b}$ shows the tangential wind speed along a radial 


$\begin{array}{cccc}\Delta / R_{1}=5 \quad t=0 h r & t=12 h r & t=12 h r & t=12 h r \\ \gamma=4 & \gamma=7 & \gamma=10\end{array}$

$\underline{r^{*}=1 / 3}$

$\alpha=0.7$
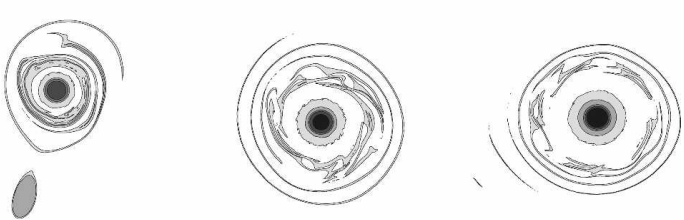

$\alpha=0.5$
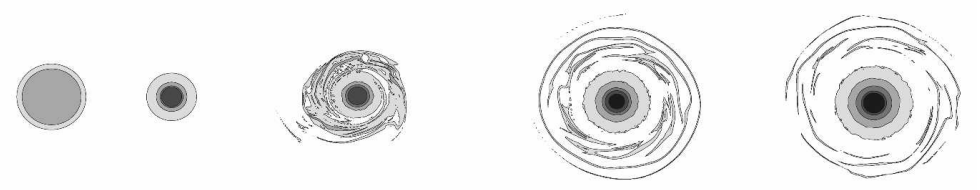

$\underline{r^{*}=1 / 4}$

$\alpha=0.7$
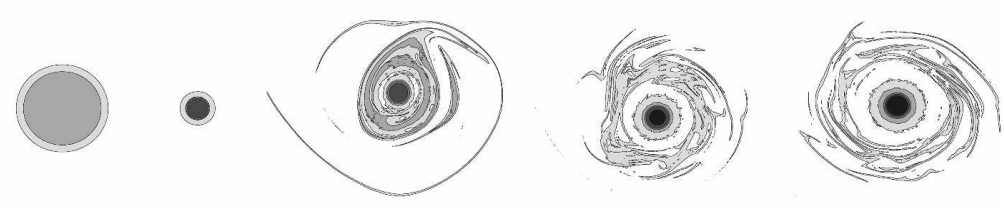

$\alpha=0.5$
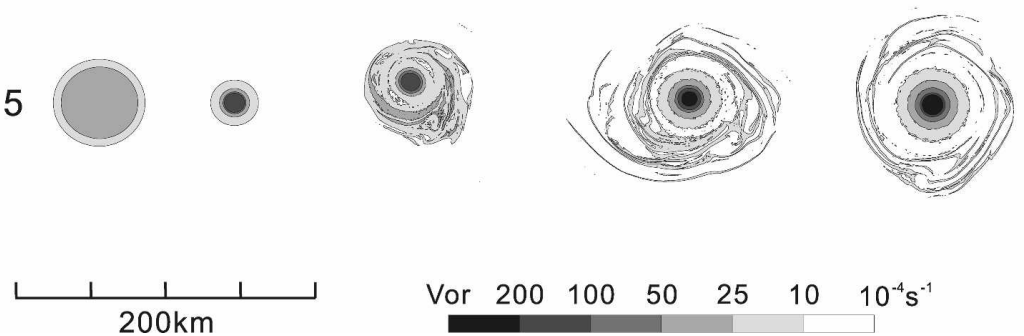

100

5025

FIG. 7. As in Fig. 6 except that the dimensionless gap parameter has been changed to $\Delta / R_{1}=5$.

emanating southward from the center of the core vortex. The wind profiles show a secondary wind maximum that apparently contracts over a period of $10 \mathrm{~h}$. During this period, the secondary wind maximum increases from $25 \mathrm{~m} \mathrm{~s}^{-1}$ at $55 \mathrm{~km}$ to $40 \mathrm{~m} \mathrm{~s}^{-1}$ at $35 \mathrm{~km}$. The overall features of the contraction in Fig. $8 \mathrm{~b}$ are in general agreement with the observations of Hurricane Gilbert $^{6}$ (Black and Willoughby 1992). Thus, while the contraction mechanism for outer bands is often argued to be an axisymmetric gradient-balanced process involving advection by the divergent part of the flow (Shapiro and Willoughby 1982), Fig. 8 suggests that vorticity advection by the nondivergent part of the flow can also play an important role.

\footnotetext{
${ }^{6}$ A discrepancy with the Gilbert observations is the slight expansion and intensification of the primary wind maximum in our model. The azimuthal average (not shown), however, indicates an insignificant change of the primary wind maximum.
}

To summarize our large set of binary vortex interaction experiments we have classified the interactions using the scheme devised by DW and extended by Prieto et al. (2003) and KLCW. The five categories are (i) elastic interaction, (ii) merger, (iii) straining out, (iv) tripole, and (v) concentric. Figure 9 shows the final $(12 \mathrm{~h})$ vorticity fields for typical examples of these five categories. The experimental parameters used in the Fig. 9 examples are given in Table 1. The difference between the concentric and straining-out regimes is determined subjectively from the width of the outer vorticity bands. Cases with multiple thin bands (typical width of $2.5-4 \mathrm{~km}$ ) are placed in the straining-out regime.

Figure 10 summarizes the binary interaction regimes as a function of the skirt parameter $\alpha$, the dimensionless gap $\Delta / R_{1}$ and the vorticity strength ratio $\gamma=\zeta_{1} / \zeta_{2}$ for the radius ratios $r^{*}=R_{1} / R_{2}=1 / 2,1 / 3$, and $1 / 4$ (Figs. $10 \mathrm{a}, \mathrm{b}, \mathrm{c}$, respectively). The abscissa in the two-dimen- 
(a)

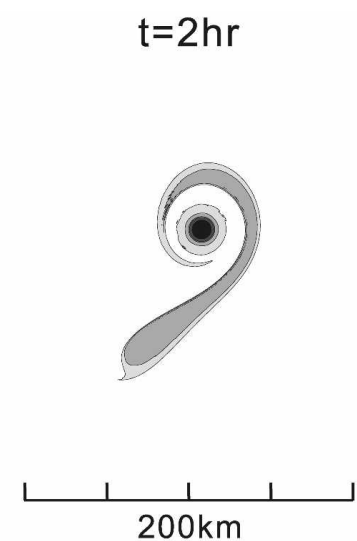

$\mathrm{t}=6 \mathrm{hr}$

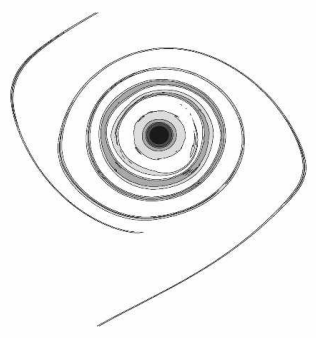

$$
\mathrm{t}=12 \mathrm{hr}
$$
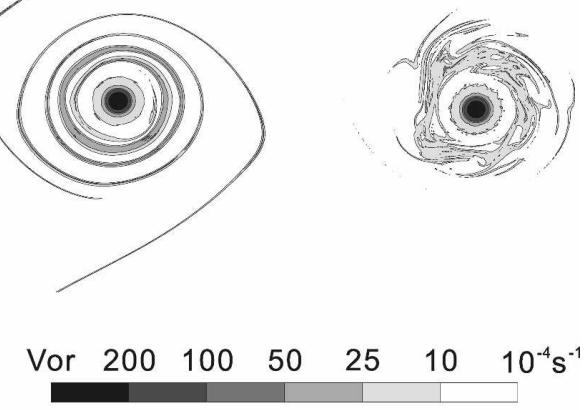

(b)

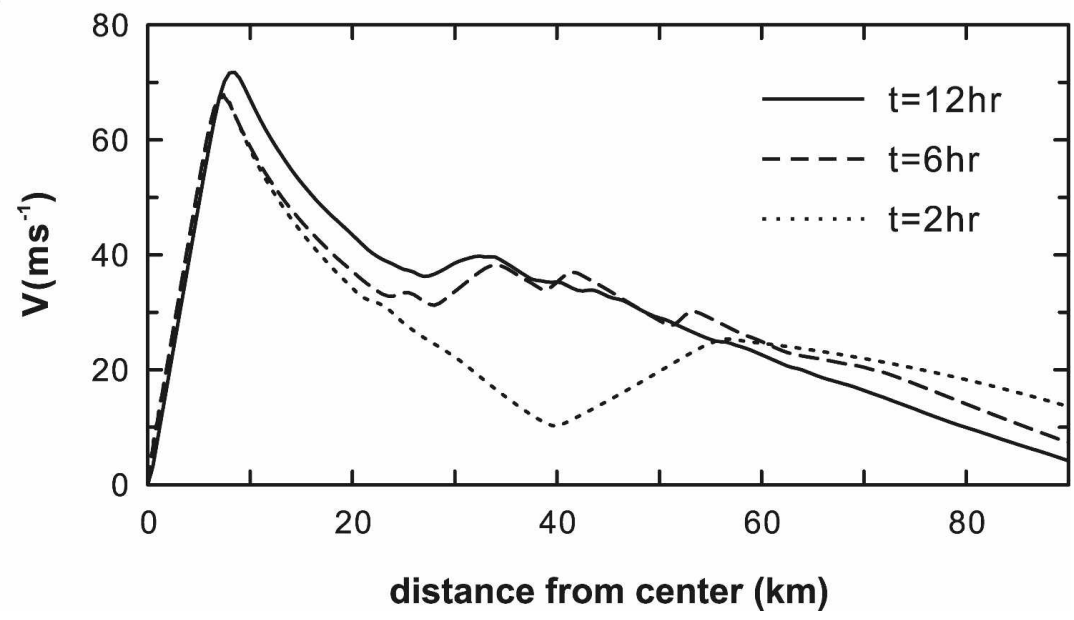

FIG. 8. (a) Vorticity fields at hours 2, 6, and 12 and (b) tangential wind speed along a radial emanating southward from the center of the strong core vortex for an experiment with $\alpha=$ $0.7, \gamma=7, r^{*}=1 / 4$, and $\Delta / R_{1}=5$.

sional parameter space (each layer of Fig. 10) is the dimensionless gap $\Delta / R_{1}$, which ranges ${ }^{7}$ from 0 to 7 . The ordinate is the vorticity strength ratio $\gamma$, which ranges from 4 to 10 . The Rankine $(\alpha=1)$ cases are reproduced from Fig. 10 of KLCW, with the addition of the results from the $\Delta / R_{1}=5,6$ experiments. Figure 10a suggests that concentric structures in general do not form around skirted vortices $(\alpha=0.7$ and $\alpha=0.5)$ when the initial companion vortex is twice the radius of the core vortex. Elastic interactions occur for Rankine vortices in the region with large dimensionless gap (i.e., $\Delta / R_{1} \geq$ 4 ), while the companion vortex tends to be strained out for skirted vortices $(\alpha=0.7$ and $\alpha=0.5)$. Figures 10b,c indicate that narrow-skirted vortices $(\alpha=0.7)$ mainly produce concentric structures for $\Delta / R_{1} \geq 2$ for $r^{*}=1 / 4$

\footnotetext{
${ }^{7}$ We have also performed experiments with $\Delta / R_{1}=8$ and found no concentric end states.
}

and $\Delta / R_{1} \geq 3$ for $r^{*}=1 / 3$. Wide-skirted vortices $(\alpha=$ $0.5)$ produce concentric structures for $\Delta / R_{1} \geq 4$, but result in the straining-out regime when the core vortex is sufficiently strong (i.e., $\gamma$ is large). It is particularly interesting that the formation of concentric vorticity structures for a wide-skirted vortex $(\alpha=0.5)$ requires a separation distance that is at least 4 times the core vortex radius. The Rankine vortex produces a concentric vorticity structure when the separation distance is less than 3 or 4 times the core vortex radius. In general, when the companion vortex is large $\left(r^{*} \leq 1 / 3\right)$, skirted vortices produce concentric structures in the region where the Rankine vortex has elastic interaction (i.e., $\left.\Delta / R_{1} \geq 4\right)$.

To summarize, the regime diagrams presented in this section indicate that a Rankine vortex favors the formation of a concentric structure closer to the core vortex, while skirted vortices promote the formation of concentric structures farther from the core vortex. 


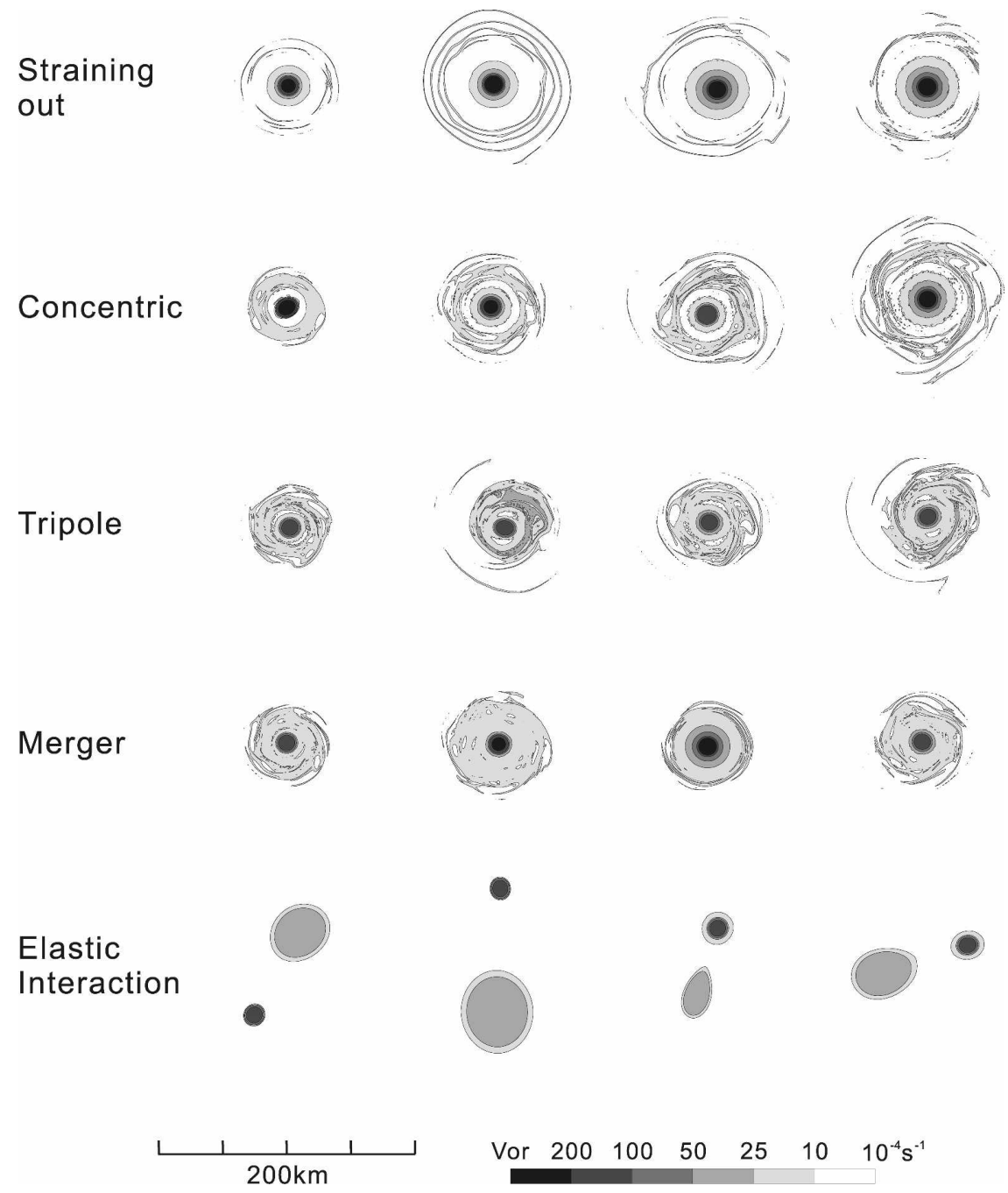

FIG. 9. Examples of the vorticity field at hour 12, showing different classifications of binary vortex interactions involving a skirted core vortex. See Table 1 for experimental parameters.

Thus, it is conceivable that a core vortex of sufficient strength can form a concentric vorticity structure at large radius through binary vortex interaction. This is consistent with satellite microwave observations that suggest a wide range of diameters for concentric eyewalls.

\section{Summary and concluding remarks}

We have studied the effect of vorticity skirts on the formation of concentric vorticity structures in binary vortex interactions. Our results indicate several requirements that must be met in order to form concentric structures from binary interactions. (i) The small vortex must be at least 4-6 times as strong as the larger companion vortex. (ii) The separation distance be- tween the edges of the two vortices must also be less than 6-7 times the smaller vortex radius. In general, when the companion vortex is 3 times the size of the core vortex, a core vortex with a vorticity skirt produces a concentric structure if the separation distance is 4 times as great as the radius of the smaller vortex. In contrast, the Rankine vortex has elastic interactions in this region, except for the few cases with a very strong core and a fairly large companion vortex. The main point here is that when a skirted core vortex of sufficient strength interacts with a companion vortex of sufficient size, a concentric vorticity structure forms at a larger radius than is allowed by a similar, but unskirted core vortex. We have shown that concentric eyewalls of various sizes, such as those depicted in Fig. 1, can be produced through binary vortex interaction with 
TABLE 1. The experimental parameters of Fig. 9.

\begin{tabular}{|c|c|c|c|c|}
\hline \multirow[t]{4}{*}{ Straining out } & $\alpha=0.7$ & $\alpha=0.7$ & $\alpha=0.5$ & $\alpha=0.5$ \\
\hline & $\gamma=7$ & $\gamma=10$ & $\gamma=9$ & $\gamma=10$ \\
\hline & $\Delta / R_{1}=4$ & $\Delta / R_{1}=6$ & $\Delta / R_{1}=6$ & $\Delta / R_{1}=6$ \\
\hline & $r^{*}=1 / 2$ & $r^{*}=1 / 3$ & $r^{*}=1 / 3$ & $r^{*}=1 / 4$ \\
\hline \multirow[t]{4}{*}{ Concentric } & $\alpha=1.0$ & $\alpha=0.7$ & $\alpha=0.7$ & $\alpha=0.5$ \\
\hline & $\gamma=8$ & $\gamma=7$ & $\gamma=6$ & $\gamma=8$ \\
\hline & $\Delta / R_{1}=0$ & $\Delta / R_{1}=3$ & $\Delta / R_{1}=5$ & $\Delta / R_{1}=4$ \\
\hline & $r^{*}=1 / 3$ & $r^{*}=1 / 3$ & $r^{*}=1 / 4$ & $r^{*}=1 / 4$ \\
\hline \multirow[t]{4}{*}{ Tripole } & $\alpha=0.7$ & $\alpha=0.7$ & $\alpha=0.5$ & $\alpha=0.5$ \\
\hline & $\gamma=4$ & $\gamma=4$ & $\gamma=4$ & $\gamma=4$ \\
\hline & $\Delta / R_{1}=3$ & $\Delta / R_{1}=4$ & $\Delta / R_{1}=4$ & $\Delta / R_{1}=4$ \\
\hline & $r^{*}=1 / 3$ & $r^{*}=1 / 4$ & $r^{*}=1 / 3$ & $r^{*}=1 / 4$ \\
\hline \multirow[t]{4}{*}{ Merger } & $\alpha=0.7$ & $\alpha=0.7$ & $\alpha=0.5$ & $\alpha=0.5$ \\
\hline & $\gamma=4$ & $\gamma=7$ & $\gamma=10$ & $\gamma=4$ \\
\hline & $\Delta / R_{1}=2$ & $\Delta / R_{1}=1$ & $\Delta / R_{1}=3$ & $\Delta / R_{1}=3$ \\
\hline & $r^{*}=1 / 3$ & $r^{*}=1 / 4$ & $r^{*}=1 / 2$ & $r^{*}=1 / 3$ \\
\hline \multirow{4}{*}{$\begin{array}{l}\text { Elastic } \\
\text { interaction }\end{array}$} & $\alpha=1.0$ & $\alpha=1.0$ & $\alpha=0.7$ & $\alpha=0.7$ \\
\hline & $\gamma=4$ & $\gamma=4$ & $\gamma=4$ & $\gamma=4$ \\
\hline & $\Delta / R_{1}=5$ & $\Delta / R_{1}=6$ & $\Delta / R_{1}=6$ & $\Delta / R_{1}=6$ \\
\hline & $r^{*}=1 / 3$ & $r^{*}=1 / 4$ & $r^{*}=1 / 2$ & $r^{*}=1 / 3$ \\
\hline
\end{tabular}

skirted vorticity outside the cyclone core. Future work is needed to determine how moist convection modifies these results.

Our approach implicitly assumes that moist convection, such as observed in Fig. 1, is a signature of enhanced vorticity. It is reasonable to expect that the convergence term in the vorticity equation allows vorticity to be generated by the lower-tropospheric convergence associated with convection in the vicinity of the vortex core. Moreover, RSMK implies that patches of vorticity and convection are most likely to be collocated if the convection survives the hostile environment of the strain-dominated rapid filamentation zone. There is evidence that convection and vorticity are highly correlated in real storms. For example, Simpson et al. (1997) reported an elastic interaction (mutual rotation) of two 100-km-scale convective systems and Hendricks et al. (2004) reported a "vortical hot tower" of deep convection that had a scale of several tens of kilometers. Zhang et al. (2005) also found enhanced vorticity associated with convective bands in fifth-generation Pennsylvania State University-National Center for Atmospheric Research Mesoscale Model (MM5) simulations of the concentric eyewalls of Typhoon Winnie.

Because tropical cyclones often weaken after a concentric eyewall forms, intensity forecasting could obviously benefit from an improved prediction of this process. Our argument suggests that it may be important to observe and understand the spatial and temporal characteristics of the vorticity field outside the cyclone core, as well as the detailed core structure, in order to better predict the formation of concentric eyewalls. The present study treats convection in the cyclone environment as an existing companion vortex. The vorticitygenerating processes that determine the scale and strength of vorticity in the cyclone environment require further exploration. In this regard, several studies have described the vorticity/convection initiation processes that can occur in concert with the organizational dynamics we have discussed. For example, Montgomery and Kallenbach (1997) suggest that the absorption of radially propagating linear vortex Rossby waves at a critical radius outside the RMW may be an important mechanism for the formation of concentric eyewalls. Nong and Emanuel (2003) discuss the formation of concentric eyewalls in their axisymmetric model as a finiteamplitude Wind-induced Surface Heat Exchange (WISHE) instability, possibly triggered by upper-level external forcing.

It is interesting to note that the appearance of tripoles in the present study adds to a long list of methods by which these structures can be produced. For example, tripoles emerge from unstable initial states in laboratory experiments with both rotating fluids (Kloosterziel and van Heijst 1991; van Heijst et al. 1991; Denoix et al. 1994) and pure electron plasmas (Driscoll and Fine 1990), as coherent structures in twodimensional turbulence simulations (Legras et al. 1988), as the result of collisions of two dipoles (Larichev and Reznik 1983; Orlandi and van Heijst 1992), as a result of finite-amplitude quadrapolar (i.e., azimuthal wavenumber 2) distortions of a monopolar Gaussian vorticity distribution (Rossi et al. 1997), as a result of the barotropic instability across the annular region separating a strong core vortex from a weaker vorticity band (Kossin et al. 2000), and as the end state of an initial vorticity distribution in which a low vorticity eye is uncentered within an eyewall region of high vorticity (Prieto et al. 2001). Given their robustness and multitude of production methods, we might ask the question: Do tripoles actually play a role in tropical cyclone dynamics? Unfortunately, current in situ measurement techniques, which involve aircraft flight legs in only a few radial directions, do not provide sufficient data to compute vorticity fields that would reveal a tripole structure even if it were present. Observations with increased azimuthal resolution would permit finer-scale analyses of vorticity and potential vorticity. Perhaps airborne Doppler radar, such as the one used in the Hurricane Rainband and Intensity Change Experiment (RAINEX) experiment (Houze et al. 2007), will eventually provide such data. In any event, because tripole structures are so robust and can be produced in so many different ways, it would be surprising if they did not play some role in tropical cyclone dynamics. If they 
(a)
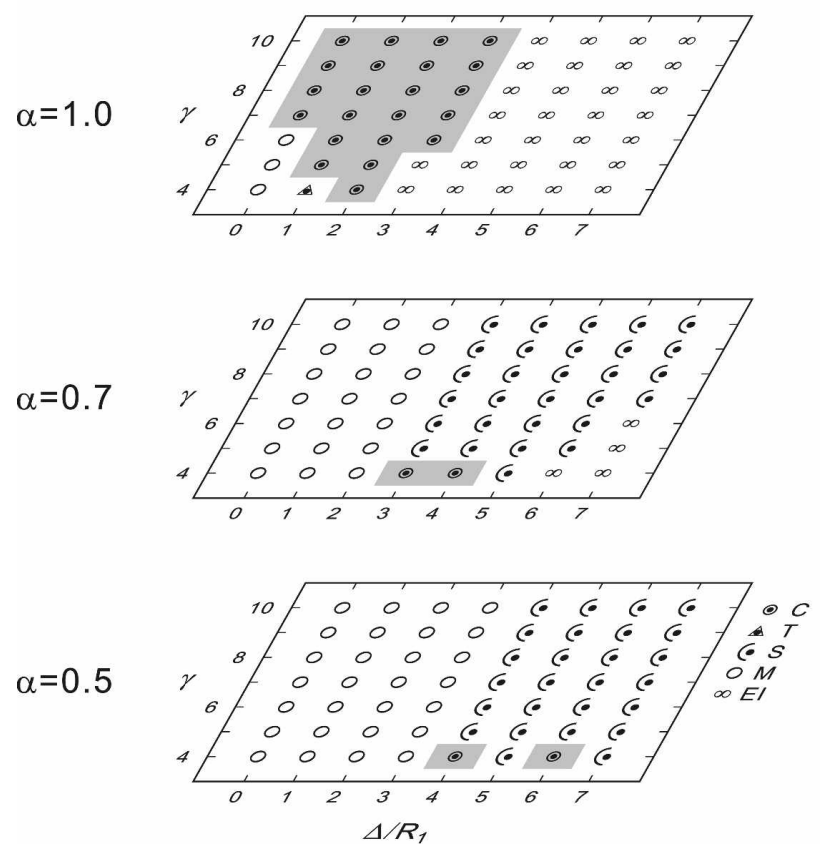

(c)
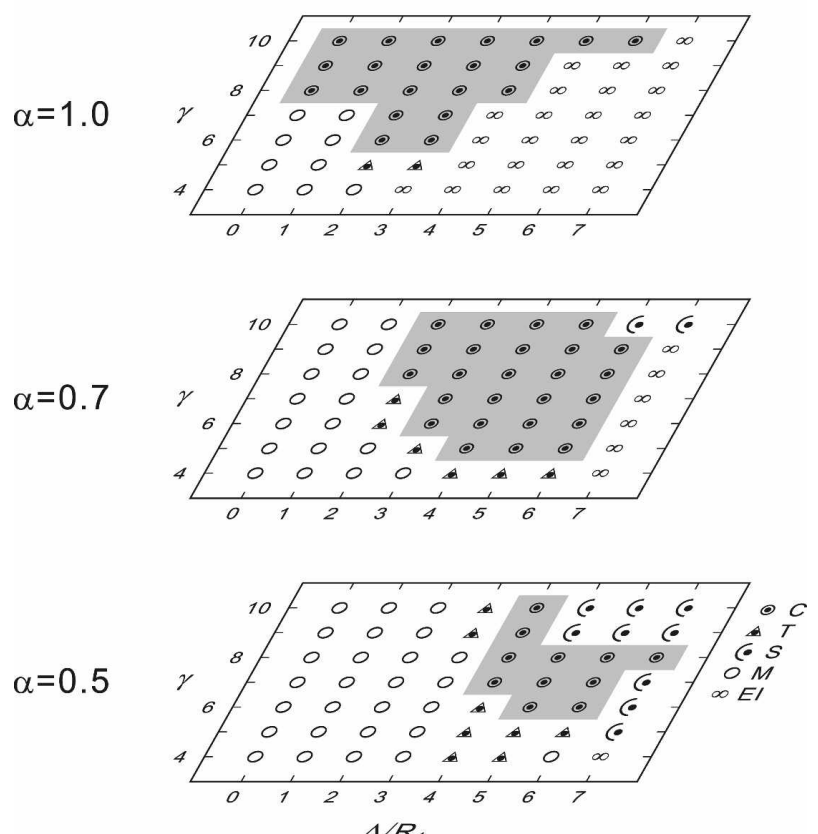

$\Delta / R_{1}$

are eventually observed in hurricanes, it will be an indication of incomplete mixing in the hurricane core, since, from statistical mechanics arguments, tripoles are a restricted statistical equilibrium far from the limit of strong mixing (Robert and Rosier 1997; Chavanis and Sommeria 1998). The phenomenon of incomplete mixing occurs in many of our simulations, including those (b)

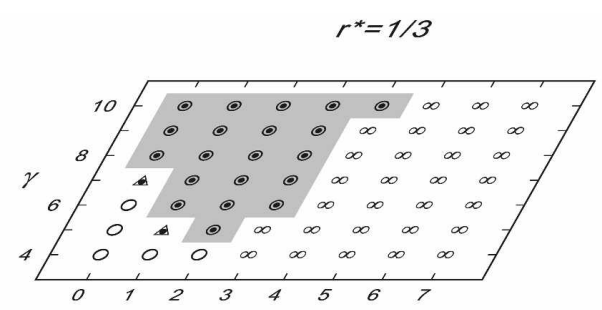

$\alpha=0.7$

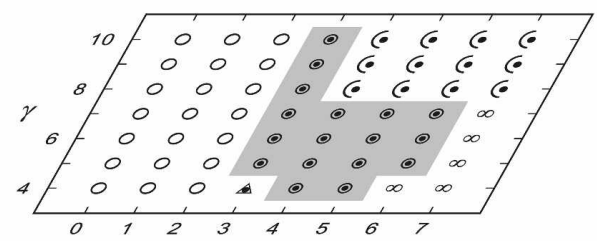

$\alpha=0.5$

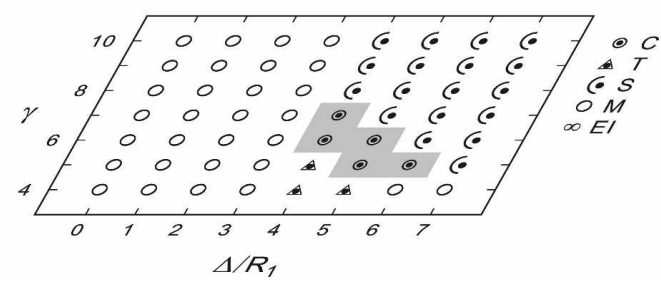

FIG. 10. Summary of the binary interaction regimes as a function of the skirt parameter $\alpha$, the dimensionless gap $\Delta / R_{1}$, and the vorticity strength ratio $\gamma=\zeta_{1} / \zeta_{2}$ for the radius ratios $r^{*}=R_{1} / R_{2}=$ (a) $1 / 2$, (b) $1 / 3$, and (c) $1 / 4$. As indicated by the code at the lower right, the structures are categorized as follows: elastic interaction (EI), merger (M), straining out (S), tripole (T), and concentric (C). The concentric cases are shaded for clarity. simulations in which tripole structures evolve; this covers the region of parameter space separating the merger and the concentric regimes.

In closing, we emphasize that a complete theory of the life cycle of concentric eyewalls, which occur in a highly convective, cloudy, precipitating atmosphere, should obviously be based on a "full-physics" model. 
However, certain aspects of the problem can be simulated using simpler models. Here our intention has been modest and has involved the use of the simplest dry dynamical arguments (based on the nondivergent barotropic model) to aid in understanding the organizational aspects of outer eyewall formation from asymmetric patterns of vorticity outside the cyclone core. It is encouraging to note that the concentric eyewall phenomenon is now being studied with more complex models and with modern aircraft instrumentation. For example, in a recent study with a full-physics tropical cyclone model, Wang (2008) has found that the rapid filamentation process can effectively suppress highazimuthal wavenumber asymmetries immediately outside the radius of maximum wind but has little effect on well-organized low wavenumber spiral bands. In addition, Houze et al. (2007) have presented observations that illustrate how the temperature and moisture profiles in the moat can evolve into a vertical structure closely resembling that found in the eye. These observations highlight the important role of subsidence in concentric eyewall cycles. Together, the work of Wang (2008), Houze et al. (2007), and Schecter and Montgomery (2007) reveal the limitations of the barotropic idealization: while it can yield insight into some of the organizational aspects of concentric eyewall cycles, it falls well short of a complete theory because of its neglect of vertical motion, the frictional boundary layer, and diabatic processes.

Acknowledgments. We thank Brian McNoldy, Chris Rozoff, Michael Montgomery, Jeffrey Hawkins, James Kossin, Lee-Yaw Lin, and Jonathan Vigh for their helpful comments and suggestions. This research was supported by the National Research Council of Taiwan through Grants NSC96-2111-M-002-002, NSC95-2745P-002-004, and MOTC-CWB-96-2M-01 to National Taiwan University, and by NASA/TCSP Grant 040007-0031 to Colorado State University.

\section{REFERENCES}

Balmforth, N. J., S. G. Llewellyn Smith, and W. R. Young, 2001: Disturbing vortices. J. Fluid Mech., 426, 95-133.

Black, M. L., and H. E. Willoughby, 1992: The concentric eyewall cycle of Hurricane Gilbert. Mon. Wea. Rev., 120, 947-957.

Carton, X., and B. Legras, 1994: The life-cycle of tripoles in twodimensional incompressible flows. J. Fluid Mech., 267, 53-82.

— , G. R. Flierl, and L. M. Polvani, 1989: The generation of tripoles from unstable axisymmetric isolated vortex structure. Europhys. Lett., 9, 339-344.

Chavanis, P. H., and J. Sommeria, 1998: Classification of robust isolated vortices in two-dimensional hydrodynamics. J. Fluid Mech., 356, 259-296.
Cushman-Roisin, B., 1994: Introduction to Geophysical Fluid Dynamics. Prentice Hall, 320 pp.

DeMaria, M., and J. C. L. Chan, 1984: Comments on "A numerical study of the interactions between two tropical cyclones." Mon. Wea. Rev., 112, 1643-1645.

Denoix, M.-A., J. Sommeria, and A. Thess, 1994: Twodimensional turbulence: The prediction of coherent structures by statistical mechanics. Progress in Turbulence Research, H. Branover and Y. Unger, Eds., AIAA, 88-107.

Driscoll, C. F., and K. S. Fine, 1990: Experiments on vortex dynamics in pure electron plasmas. Phys. Fluids, 2, 1359-1366.

Dritschel, D. G., 1989: On the stabilization of a two-dimensional vortex strip by adverse shear. J. Fluid Mech., 206, 193-221.

_ , and D. W. Waugh, 1992: Quantification of the inelastic interaction of unequal vortices in two-dimensional vortex dynamics. Phys. Fluids, 4A, 1737-1744.

Eckart, C., 1948: An analysis of the stirring and mixing processes in incompressible fluids. J. Mar. Res., 7, 265-275.

Hawkins, J. D., T. F. Lee, J. Turk, C. Sampson, J. Kent, and K. Richardson, 2001: Real-time internet distribution of satellite products for tropical cyclone reconnaissance. Bull. Amer. Meteor. Soc., 82, 567-578.

—-, M. Helveston, T. F. Lee, F. J. Turk, K. Richardson, C. Sampson, J. Kent, and R. Wade, 2006: Tropical cyclone multiple eyewall configurations. Preprints, 27th Conf. on Hurricanes and Tropical Meteorology, Monterey, CA, Amer. Meteor. Soc., 6B.1. [Available online at http://ams.confex.com/ ams/pdfpapers/108864.pdf.]

Hendricks, E. A., M. T. Montgomery, and C. A. Davis, 2004: The role of "vortical" hot towers in the formation of tropical cyclone Diana (1984). J. Atmos. Sci., 61, 1209-1232.

Houze, R. A., S. S. Chen, B. F. Smull, W.-C. Lee, and M. M. Bell, 2007: Hurricane intensity and eyewall replacement. Science, 315, 1235-1239.

Khain, A., I. Ginis, A. Falkovich, and M. Frumin, 2000: Interaction of binary tropical cyclones in a coupled tropical cycloneocean model. J. Geophys. Res., 105 (D17), 22 337-22 354.

Kloosterziel, R. C., and G. J. F. van Heijst, 1991: An experimental study of unstable barotropic vortices in a rotating fluid. $J$. Fluid Mech., 223, 1-24.

_ , and G. F. Carnevale, 1999: On the evolution and saturation of instabilities of two-dimensional isolated circular vortices. $J$. Fluid Mech., 388, 217-257.

Kossin, J. P., and W. H. Schubert, 2003: Diffusion versus advective rearrangement of a circular vortex sheet. J. Atmos. Sci., 60, 586-589.

,-- , and M. T. Montgomery, 2000: Unstable interactions between a hurricane's primary eyewall and a secondary ring of enhanced vorticity. J. Atmos. Sci., 57, 3893-3917.

Kuo, H.-C., R. T. Williams, and J.-H. Chen, 1999: A possible mechanism for the eye rotation of Typhoon Herb. J. Atmos. Sci., 56, 1659-1673.

— G. T.-J. Chen, and C.-H. Lin, 2000: Merger of tropical cyclones Zeb and Alex. Mon. Wea. Rev., 128, 2967-2975.

— L.-Y. Lin, C.-P. Chang, and R. T. Williams, 2004: The formation of concentric vorticity structures in typhoons. J. Atmos. Sci., 61, 2722-2734.

Lander, M., and G. J. Holland, 1993: On the interaction of tropical-cyclone-scale vortices. I: Observations. Quart. J. Roy. Meteor. Soc., 119, 1347-1361.

Larichev, V. D., and G. M. Reznik, 1983: On collisions between two-dimensional solitary Rossby waves. Oceanology, 23, 545552. 
Larson, R. N., 1975: Picture of the month-Hurricane twins over the eastern North Pacific Ocean. Mon. Wea. Rev., 103, 262265.

Legras, B., P. Santangelo, and R. Benzi, 1988: High resolution numerical experiments for forced two-dimensional turbulence. Europhys. Lett., 5, 37-42.

Mallen, K. J., M. T. Montgomery, and B. Wang, 2005: Reexamining the near-core radial structure of the tropical cyclone primary circulation: Implications for vortex resiliency. J. Atmos. Sci., 62, 408-425.

Matthaeus, W. H., and D. Montgomery, 1980: Selective decay hypothesis at high mechanical and magnetic Reynolds numbers. Ann. N.Y. Acad. Sci., 357, 203-222.

McWilliams, J. C., 1984: The emergence of isolated coherent vortices in turbulent flow. J. Fluid Mech., 146, 21-43.

Melander, M. V., N. J. Zabusky, and A. S. Styczek, 1986: A moment model for vortex interactions of the two-dimensional Euler equations. Part 1. Computational validation of a Hamiltonian elliptical representation. J. Fluid Mech., 167, 95 115.

Montgomery, M. T., and R. J. Kallenbach, 1997: A theory for vortex Rossby-waves and its application to spiral bands and intensity changes in hurricane. Quart. J. Roy. Meteor. Soc., 123, $435-465$.

Nong, S., and K. A. Emanuel, 2003: A numerical study of the genesis of concentric eyewalls in hurricane. Quart. J. Roy. Meteor. Soc., 129, 3323-3338.

Oda, M., M. Nakanishi, and G. Naito, 2006: Interaction of an asymmetric double vortex and trochoidal motion of a tropical cyclone with the concentric eyewall structure. J. Atmos. Sci. 63, 1069-1081.

Orlandi, P., and G. J. F. van Heijst, 1992: Numerical simulations of tripolar vortices in 2d flow. Fluid Dyn. Res., 9, 179-206.

Polvani, L. M., and X. J. Carton, 1990: The tripole: A new coherent vortex structure of incompressible two-dimensional flows. Geophys. Astrophys. Fluid Dyn., 51, 87-102.

Prieto, R., J. P. Kossin, and W. H. Schubert, 2001: Symmetrization of lopsided vorticity monopoles and offset hurricane eyes. Quart. J. Roy. Meteor. Soc., 127, 1-17.

- B. D. McNoldy, S. R. Fulton, and W. H. Schubert, 2003: A classification of binary tropical-cyclone-like vortex interactions. Mon. Wea. Rev., 131, 2656-2666.
Reasor, P. D., M. T. Montgomery, F. D. Marks, and J. F. Gamache, 2000: Low-wavenumber structure and evolution of the hurricane inner core observed by airborne dual-Doppler radar. Mon. Wea. Rev., 128, 1653-1680.

,-- , and L. D. Grasso, 2004: A new look at the problem of tropical cyclones in vertical shear flow: Vortex resiliency. $J$. Atmos. Sci., 61, 3-22.

Robert, R., and C. Rosier, 1997: The modeling of small scales in two-dimensional turbulent flows: A statistical mechanics approach. J. Stat. Phys., 86, 481-515.

Rossi, L. F., J. F. Lingevitch, and A. J. Bernoff, 1997: Quasisteady monopole and tripole attractors for relaxing vortices. Phys. Fluids, 9, 2329-2338.

Rozoff, C. M., W. H. Schubert, B. D. McNoldy, and J. P. Kossin, 2006: Rapid filamentation zones in intense tropical cyclones. J. Atmos. Sci., 63, 325-340.

Salmon, R., 1998: Lectures on Geophysical Fluid Dynamics. Oxford University Press, $378 \mathrm{pp}$.

Schecter, D. A., and M. T. Montgomery, 2007: Waves in a cloudy vortex. J. Atmos. Sci., 64, 314-337.

Shapiro, L. J., and H. E. Willoughby, 1982: The response of balanced hurricanes to local sources of heat and momentum. $J$. Atmos. Sci., 39, 378-394.

Shea, D. J., and W. M. Gray, 1973: The hurricane's inner core region. I. Symmetric and asymmetric structure. J. Atmos. Sci., 30, 1544-1564.

Simpson, J., E. A. Ritchie, G. J. Holland, J. Halverson, and S. Stewart, 1997: Mesoscale interactions in tropical cyclone genesis. Mon. Wea. Rev., 125, 2643-2661.

Terwey, W. D., and M. T. Montgomery, 2008: Secondary eyewall formation in two idealized, full-physics modeled hurricanes. J. Geophys. Res., 113, D12112, doi:10.1029/2007JD008897.

van Heijst, G. J. R., R. C. Kloosterziel, and C. W. M. Williams, 1991: Laboratory experiments on the tripolar vortex in a rotating fluid. J. Fluid Mech., 225, 301-331.

Wang, Y., 2008: Rapid filamentation zone in a numerically simulated tropical cyclone. J. Atmos. Sci., 65, 1158-1181.

Zhang, Q.-H., S.-J. Chen, Y.-H. Kuo, K.-H. Lau, and R. A. Anthes, 2005: Numerical study of a typhoon with a large eye: Model simulation and verification. Mon. Wea. Rev., 133, 725742 . 